\title{
Biophysical Properties of Magnetized Distilled Water
}

\author{
I. H. Ibrahim \\ Physics Department- Faculty of Science- Ain Shams University \\ Cairo- Egypt
}

The effect of static magnetic field on the biophysical properties of distilled water is investigated in this work. Magnetic field was applied in a direction perpendicular to distilled water drops coming from a burette (drop by drop), using a variable gap magnet, producing a magnetic field of strength varying from 0.2 up to $5 \mathrm{kG}$. Variations in the rate of flow of water, electric conductivity and dielectric constant were observed. Increasing the strength of magnetic field decreases the rate of flow, increasing both the electric conductivity and the dielectric constant of water.

\section{Introduction:}

Water is an unusual substance, mostly due to its 3D network of hydrogen bond in the molecule. Its properties allow it to act as a solvent, as a reactant, as a molecule with a cohesive properties, as an environment and as a temperature stabilizer. It has relatively high melting and boiling points for a small molecule, high specific heat capacity, and higher density of liquid than that of solid [1]. Because of water's strong hydrostatic forces water is incompressible, a property that allows cells filled with water to become turgid. Enhancement of the formation of hydrogen bonds in water molecules under high pressure was reported [2]. These unique properties of water make it perhaps the most biologically important substance. In living organisms, water is essential to solubilize and modify the properties of biomolecules such as nucleic acids, proteins and carbohydrates by forming hydrogen bonds with their polar functional groups [3].

Water is diamagnetic, however, its biophysical properties can be affected by magnetic field [4, 5]. Nakagawa et al. [6] observed magneto enhancement of water vaporization rate and attributed this to the indirect cause of frequently field effects on living organisms. Water structure, under the action of weak varying magnetic fields was investigated by the methods of absorption 
and luminescent spectroscopy [7], in which the changes in water structure is due to the clusters presented in water which control the optical heterogeneity of the medium.

Iwasaka and Ueno [8] discovered that the near IR spectrum (900-2000 $\mathrm{nm}$ ) and refractive index of water were affected by a strong magnetic field (up to $14 \mathrm{~T}$ ) and reported that, there is a possibility that the magnetic field affects the formation of hydrogen bonds of water molecules. Inaba et al. [9] suggested that the observed magnetic effects on water molecules may somehow be due to that the magnetic field strengthens water hydrogen bonding, but they commented that the exact mechanism behind these results remains a mystery.

The aim of the present work is to investigate the effect of static magnetic field of different strengths up to $5 \mathrm{kG}$ on the biophysical properties of distilled water.

\section{Materials and Methods:}

\subsection{Magnetic field:}

The magnetic field was applied using E.M -8641 variable gap magnet consists of two 3/4 inch diameter neodymium magnets on an iron .The gap may be varied from $0.5-9 \mathrm{~cm}$ to produce a magnetic field of varying strength from 0.2 up to $5 \mathrm{kG}$.

The magnetic field strength varies approximately as shown in Fig. (1), which represents the calibration curve of the magnet as a relation between the magnetic field strength at the mid point between poles and the distance between the two poles. The magnetic field strength at the mid point between poles was measured using, SE - 8606 A digital Gauss/Tesla meter.

\subsection{Water Magnetization and Rate of Flow:}

To magnetize water, the magnetic field was applied to distilled water drops coming from a burette (drop by drop), intersecting the applied field and collected in a beaker. For the determination of the rate of flow (Q) of normal and magnetized water, a stop watch was used to measure the time of flow (dt) of the measured amount of water (dw) through the gap before and after field application, then the rate of water flow can be determined $(\mathrm{Q}=\mathrm{dw} / \mathrm{dt})$ in each case. The process was repeated six times for each experiment at magnetic field strengths 1.44, 2.29, 3.14,4 and $5 \mathrm{kG}$. 


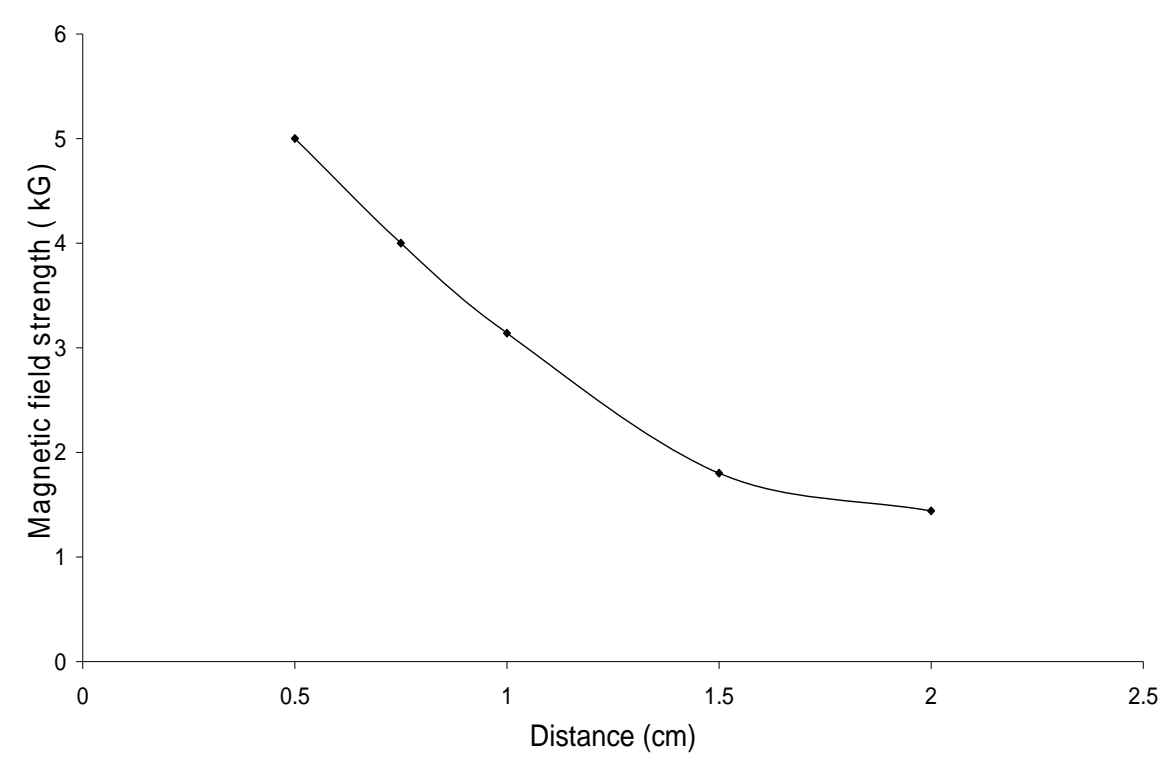

Fig. (1): The calibration curve of the permanent variable gap magnet.

\subsection{Electric conductivity and dielectric constant:}

The conductivity of normal and magnetized water was measured using a conductance bridge PJK- 301- 010X, with a glass tube filled with water of each type, this tube contains two platinum electrodes separated by a fixed distance. Before applying magnetic field, the rate of flow of water drops was fixed from the burette and water drops coming from it were collected for 5 minutes and the conductivity of collected water $\sigma_{0}$ (control) was measured. At the same rate of water flow, the magnetic fields $(1.44,2.29,3.14$, 4 or $5 \mathrm{kG}$ ) were applied to the water drops coming from the burette.

After exposure to each magnetic field strength, the electric conductivity $(\sigma)$ of the collected water (in 5 minutes) was measured with 5 minutes interval during one hour. The relative conductivity $\sigma / \sigma_{0}$ for magnetized water is calculated for each reading.

The dielectric constant $\varepsilon^{\backslash}$ was measured for control and magnetized samples using RLC bridge (universal bridge type LCR-6). The capacitance of the sample was measured at frequency $50 \mathrm{~Hz}$ and the dielectric constant was calculated for different magnetic field strengths.

Results are expressed as mean \pm S.D of 6 independent determinations. 


\section{Results and Discussion:}

The flow rate of distilled water before application of magnetic field, was found to be about $19.5 \pm 3.1 \mathrm{ml} / \mathrm{min}$. Applying magnetic field of strength $1.44 \mathrm{kG}$, there is no change in the rate of flow of water. Increasing the strength of magnetic field, the flow rate of water decreases, as shown in Fig.(2). The variations in relative conductivity at different exposure periods to magnetic field of different strengths are shown in Table (1) and Figs. (3, 4).

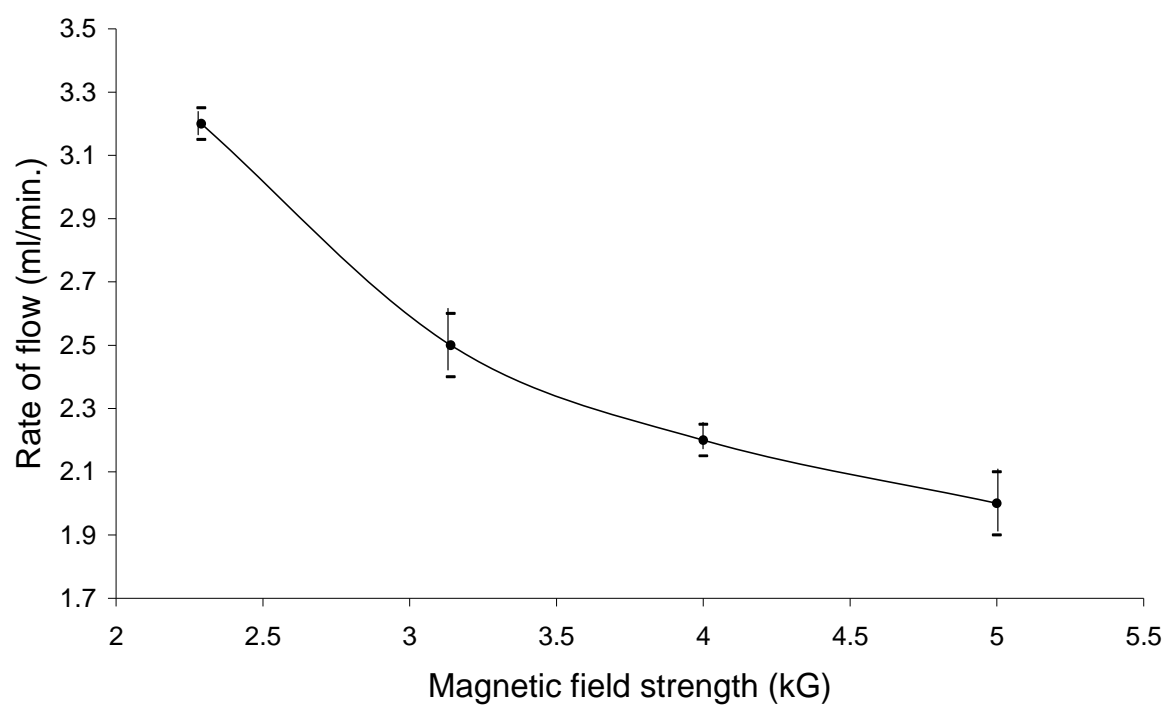

Fig (2): The relation between magnetic field strength and the flow rate of water.

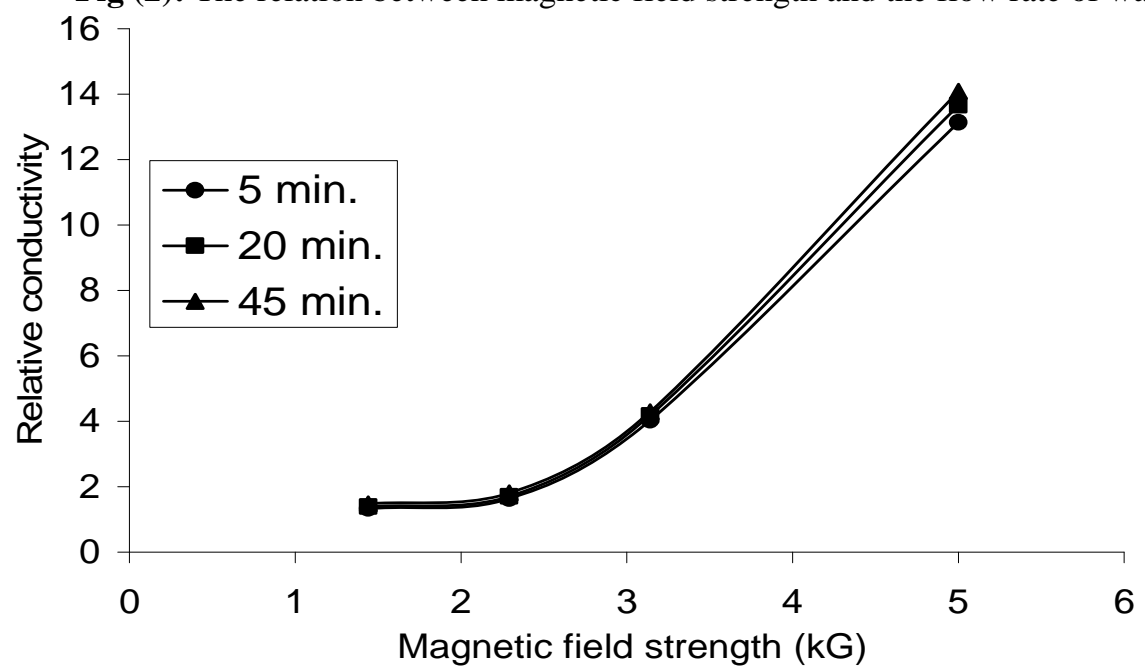

Fig.(3): Variation of the relative conductivity with the magnetic field strength as a funct the exposure time. 


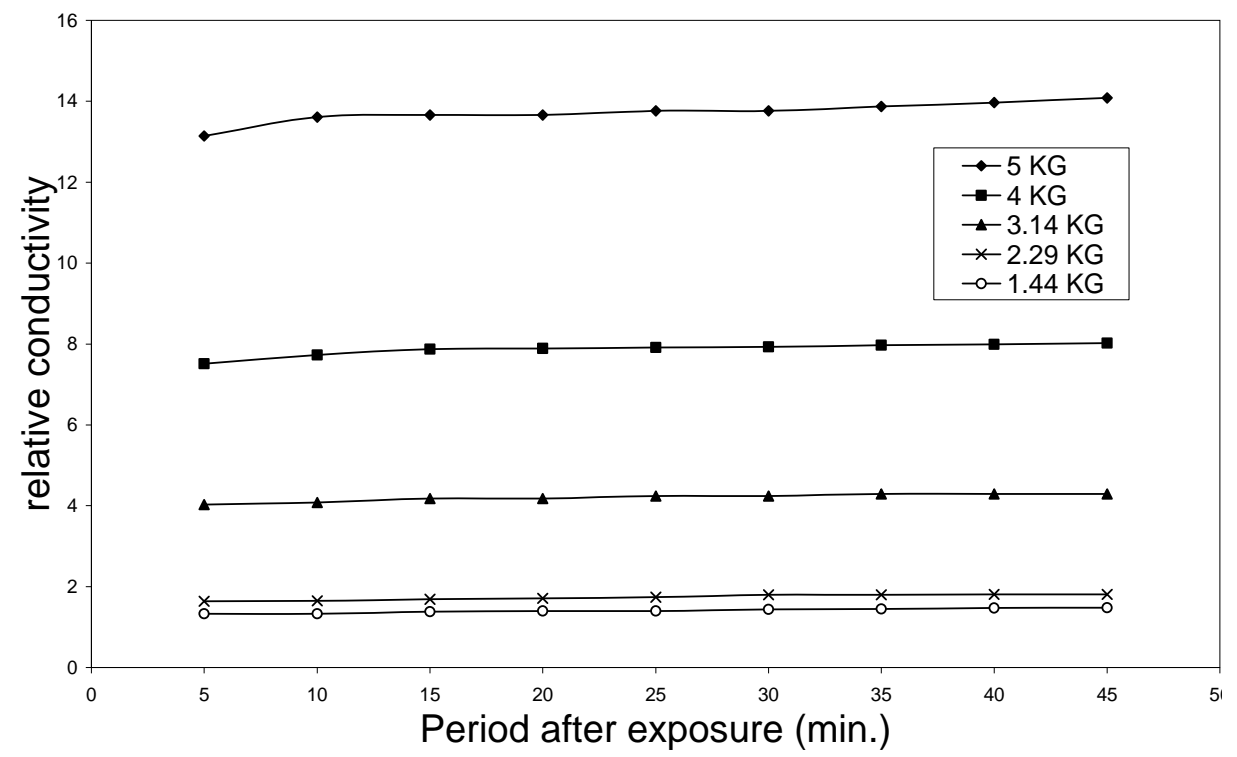

Fig.(4): Variation of the relative conductivity with the exposure time as a function of thı magnetic field strength.

Table (1): The variations in relative conductivity at different exposure periods to magnetic fields of different strengths.

\begin{tabular}{|c||c|c|c|c|c||}
\hline \multirow{2}{*}{$\begin{array}{c}\text { Period } \\
\text { after } \\
\begin{array}{c}\text { Exposure } \\
\text { (min.) }\end{array}\end{array}$} & $\begin{array}{c}\text { Strength= } \\
1.44 \mathrm{kG}\end{array}$ & $\begin{array}{c}\text { Strength= } \\
2.29 \mathrm{kG}\end{array}$ & $\begin{array}{c}\text { Strength= } \\
3.14 \mathrm{kG}\end{array}$ & $\begin{array}{c}\text { Strength }= \\
4 \mathrm{kG}\end{array}$ & $\begin{array}{c}\text { Strength } \\
5 \mathrm{kG}\end{array}$ \\
\hline \hline 5 & $1.33 \pm 0.08$ & $1.64 \pm 0.12$ & $4.03 \pm 0.60$ & $7.51 \pm 1.10$ & $13.14 \pm 2.20$ \\
\hline 10 & $1.33 \pm 0.09$ & $1.65 \pm 0.15$ & $4.08 \pm 0.70$ & $7.73 \pm 1.20$ & $13.16 \pm 2.40$ \\
\hline 15 & $1.38 \pm 0.08$ & $1.69 \pm 0.14$ & $4.18 \pm 0.65$ & $7.87 \pm 1.10$ & $13.66 \pm 2.30$ \\
\hline 20 & $1.40 \pm 0.10$ & $1.71 \pm 0.14$ & $4.18 \pm 0.70$ & $7.89 \pm 1.25$ & $13.66 \pm 2.45$ \\
\hline 25 & $1.44 \pm 0.15$ & $1.80 \pm 0.15$ & $4.24 \pm 0.80$ & $7.91 \pm 1.20$ & $13.76 \pm 2.50$ \\
\hline 30 & $1.44 \pm 0.10$ & $1.80 \pm 0.16$ & $4.24 \pm 0.70$ & $7.93 \pm 1.30$ & $13.76 \pm 2.45$ \\
\hline 35 & $1.44 \pm 0.09$ & $1.80 \pm 0.15$ & $4.29 \pm 0.75$ & $7.97 \pm 1.25$ & $13.76 \pm 2.50$ \\
\hline 40 & $1.45 \pm 0.15$ & $1.81 \pm 0.17$ & $4.29 \pm 0.80$ & $7.99 \pm 1.30$ & $13.87 \pm 2.60$ \\
\hline 45 & $1.47 \pm 0.10$ & $1.81 \pm 0.16$ & $4.29 \pm 0.75$ & $8.02 \pm 1.40$ & $14.08 \pm 2.75$ \\
\hline
\end{tabular}

It is clear from the table and figures that the relative conductivity increases as the magnetic field strength increases. Also, the relative conductivity increases by increasing the exposure period to magnetic field.

The effect of magnetic field of different strengths on the dielectric constant of magnetized water is shown in Fig. (5). The figure shows that, the dielectric constant increases by increasing the magnetic field strength. 


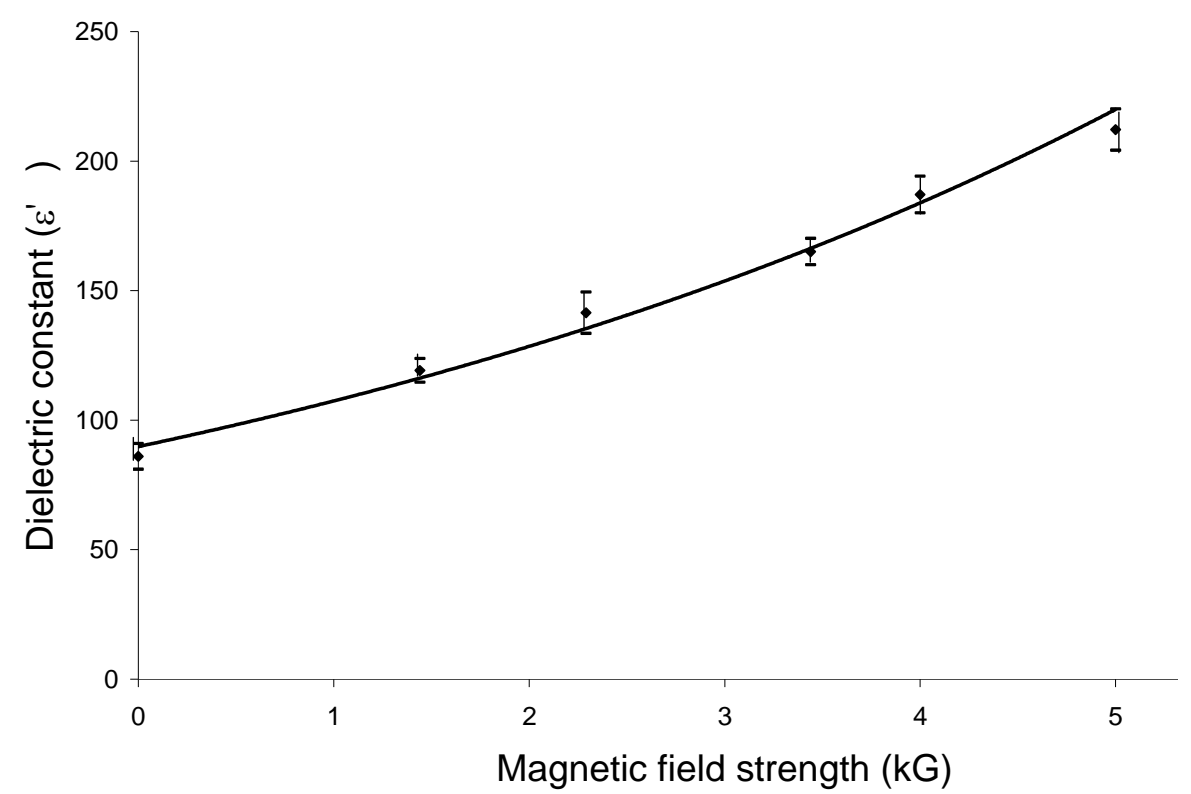

Fig.(5): Dependence of the dielectric constant of water on the magnetic field strength.

All living organisms are under the effects of artificial electric and magnetic fields, which may have either a lower or higher intensity compared to naturally occurring geomagnetic levels. The magnetic influences can be observed in different organisms both at cells and molecular level .Magnetic treatment of water clearly demonstrates that only its physical properties are modified by the effect of magnetic field, while the chemical composition and properties remain unchanged [10].

In the magnetically treated water there are maximum four hydrogen bridge connections containing protons that spin to the same direction. These are called clusters. Water that is completely organized consists of clusters. The clusters are not stable but very sensitive and unstable units. The influence of the magnetic fields on water is to change the dimensions of that clusters, which affects the physical properties of water [7].

\section{Conclusions:}

From the obtained results, it may be concluded that, application of magnetic field resists the motion of water drops, as they pass perpendicular to the direction of the applied magnetic field and may decrease the intermolecular distances between water molecules. This conclusion may explain the observed decrease in the rate of flow of water under the effect of the applied magnetic field. Application of magnetic field on water may make also alignment of water 
clusters and that alignment may be increased by increasing the magnetic field strength. The alignment of water clusters may increase its conductivity to electric current. This increase is essentially permanent and is different from original. Also it may be concluded that, the applied magnetic field may affect the formation of hydrogen bonds of water molecules and that may lead to conformational changes. These changes may be the reason for the observed variations in both conductivity and dielectric constant. This work concluded some changes in biophysical properties of water due to the effect of magnetic field and these changes were varied in accordance with the filed strength. These changes in turn may cause bioeffects, which will be our goal in future studies.

\section{References}

1. D. Eisnberg and W. Kauzmann, "The Structure and The Properties of Water”, Oxford University press (1969).

2. M. Kato, Y. Taniguchi, S. Sawamura and K. Suzuki, "Physics and Chemistry of Ice", edited by N. Maeno and T. Hondoh - Hokkaido University press (1992).

3. I. Jerman, M. Berden and R. Ruzic, Electro - Magnetobiology 15, (3), 229 (1996).

4. S. Rai, N.N. Singh and R. N. Michra, Biology Engineering Computers 32, 614 (1994).

5. S. Rai and A. K. Singh, Electro Magnetobiology, 14, 23 (1995).

6. J. Nakagawa, N. Hirota, K. Kitazawa and M. Shoda, .J. Appl. Phys., 85, 2923 (1999).

7. A. N. Baranov, V. F. Kiselev, V. V. Rozanov and A. M. Soletskii, Aviakosm. Ekolog. Med. 29 (6), 45 (1995).

8. M. Iwasaka and S. Ueno, J. Appl. Phys. 83, (11), 6459 (1998).

9. H. Inaba, T. Saitou, K. Tozaki and H. Hayashi, J. Appl. Phys. 96, (11), 6127 (2004).

10. M. Singh and K. P. Singh, J. Anat. Soc. India 51 (1), 47 (2002). 